\title{
Aves de um fragmento de Mata Atlântica no alto Rio Doce, Minas Gerais: colonização e extinção
}

\author{
Christiana M. A. Faria ${ }^{1 ;}$ Marcos Rodrigues ${ }^{1 ;}$ Frederico Q. do Amaral ${ }^{1}$; \\ Érica Módena ${ }^{1} \&$ Alexandre M. Fernandes ${ }^{2}$
}

\begin{abstract}
${ }^{1}$ Laboratório de Ornitologia, Departamento de Zoologia, Instituto de Ciências Biológicas, Universidade Federal de M inas Gerais. Caixa Postal 486, 31270-901 Belo Horizonte, Minas Gerais, Brasil. E-mail: christianafaria@yahoo.com.br; ornito@mono.icb.ufmg.br

${ }^{2}$ Coleções Zoológicas, Aves e Programa de pós-graduação em Genética,Conservação e Biologia Evolutiva, Instituto Nacional de Pesquisa da Amazônia. Caixa Postal 478, 69011-970 Manaus, Amazonas, Brasil.
\end{abstract}

\begin{abstract}
The birds of an Atlantic Forest fragment at upper Rio Doce valley, Minas Gerais, southeastern Brazil: colonization and extinction. It is presented a bird survey of a forest fragment at the upper Rio Doce valley carried out from April 2002 to November 2004. The region is within one of the most important Brazilian hot spot for biological conservation: the Atlantic Forest. The fragment is within 'Estação de Desenvolvimento Ambiental de Peti' (EPDA-Peti) under the premises of a hydroelectric power station run by the Minas Gerais Energetic Company. It holds 605 ha of a mosaic of secondary growth forest patches from different ages, characterised mainly by semi deciduous forest. Bird census were carried out through transect, mist-net captures, point counts and recording bird vocalizations. The present survey was compared to an unpublished survey performed in 1989 to find out possible extinctions and colonization. It was recorded 231 species, belonging to 57 families. This represents 33\% of all 682 bird species recorded for the Atlantic forest biome of east Brazil. Thirtythree species are considered endemic to the Atlantic Forest and one is considered endemic to the Cerrado biome of central Brazil. Five species are threatened in the state of Minas Gerais and one species is globally threatened to extinction, the red-billed curassow Crax blumenbachii Spix, 1825 (Cracidae). It was found 35 species not reported before for the area. Also, 52 species went locally extinct from the fragment. The EPDA-Peti holds a significant number of the Atlantic Forest bird species, and long-term bird monitoring on such fragments will reveal important aspects for the understanding of colonization and extinction in the biome. KEY WORDS. Avian survey; biodiversity; reintroduction; endangered species.
\end{abstract}

RESUMO. Foi conduzido um levantamento de espécies de aves em um fragmento florestal no alto Rio Doce de abril de 2002 a novembro de 2004. A região está inserida numa das áreas prioritárias para conservação da biodiversidade do Brasil, a Mata Atlântica. O fragmento está dentro da ‘Estação de Pesquisa e Desenvolvimento Ambiental de Peti' (EPDA-Peti) e cerca uma represa de uma usina hidroelétrica das 'Companhia Energética de Minas Gerais’. O fragmento possui 605 ha de mata secundária semidecídua em vários estágios de regeneração. O método utilizado foi o de observação direta ao longo de 'transectos', captura com redes, pontos de escuta e identificação a partir do uso de vocalizações. Foram registradas 231 espécies de aves pertencentes a 57 famílias. Isso corresponde cerca de $33 \%$ das 682 espécies já registradas para o bioma da Mata Atlântica. Constam nesta lista 33 espécies endêmicas da Mata Atlântica e uma endêmica do Cerrado. Cinco espécies são ameaçadas em Minas Gerais e uma espécie ameaçada de extinção global, o mutum do sudeste Crax blumenbachii Spix, 1825 (Cracidae). Foram encontradas 35 novas espécies em relação ao levantamento de 1989, bem como a extinção local de outras 52 espécies. O fragmento florestal da EPDA-Peti abriga uma porção significativa da avifauna da Mata Atlântica, e monitoramento de longo prazo irá revelar aspectos importantes para compreensão dos mecanismos de extinção e colonização na Mata Atlântica.

PALAVRAS-CHAVE. Avifauna; biodiversidade; reintrodução; espécies ameaçadas.

A Mata Atlântica pode ser considerada um dos biomas com o maior número de endemismos do planeta. Esse ecossistema apresenta muitas espécies endêmicas de vegetais
(Morl et al. 1983), insetos (Brown 1987), anfíbios (HADDAD \& SAZImA 1992), aves (Stotz et al. 1996) e mamíferos (KINSEY 1982). Porém, muitos desses organismos endêmicos se encontram 
ameaçados de extinção, pois este bioma é considerado um dos mais fragmentados e ameaçados do globo (BıвBY et al. 1992). O mesmo acontece com a sua avifauna, a qual é composta por 682 espécies, 199 das quais são endêmicas, e destas, 144 estão em perigo de desaparecer, principalmente devido à destruição do seu hábitat (Stotz et al. 1996, BirdLife International 2000). Mesmo diante desse quadro devastador, pouco se conhece sobre a avifauna remanescente nos fragmentos criados e estabelecidos nestes últimos anos (e.g. Ribon et al. 2003). O primeiro passo para que o processo de extinção e colonização seja melhor entendido, é o levantamento de espécies nestes fragmentos.

A Estação de Pesquisa e Desenvolvimento Ambiental de Peti (doravante EPDA-Peti) é hoje um fragmento florestal na bacia do alto Rio Doce, uma das regiões de Mata Atlântica mais fragmentada do sudeste do Brasil (MACHADo \& FonseCA 2000). Este fragmento florestal está isolado por uma matriz composta principalmente por antigas plantações de Eucalyptus, pastos e áreas de solo exposto devido à mineração que circunda parte da represa da Usina Hidrelétrica de Peti, administrada pela Companhia Energética de Minas Gerais (CEMIG). O pouco conhecimento que se tem sobre a diversidade de aves da EPDAPeti encontra-se em um relatório técnico que lista 248 espécies de aves. Essa lista pode ser usada como base para se estudar questões conservacionistas, como por exemplo, a extinção local ea colonização de espécies em fragmentos florestais de $M$ ata Atlântica isolados (veja, por exemplo, Rıbon et al. 2003).

Desde 1999 são realizadas campanhas de soltura de espé cies na estação, al gumas bem sucedidas e outras não. Até agora 31 espécies já foram soltas (Anexo I), de acordo com a lista cedida pelos responsáveis na EPDA-Peti.

O objetivo do presente artigo foi realizar um inventário das espécies de aves que ocorrem na mata remanescente da EPDA-Peti e avaliar a mudança na composição das espécies na área em relação ao inventário anterior.

\section{MATERIAL E MÉTODOS}

\section{Área de Estudo}

Este estudo foi conduzido na Estação de Pesquisa e Desenvolvimento Ambiental de Peti, que possui 605 ha e está localizada no município de Santa Bárbara, a 100 km de Belo Horizonte, entre as latitudes Sul de 19052"23' e 1954"27' e as longitudes Oeste de 43020"51' e 4323"28' (Fig. 1). A área possui um relevo ondulado, formado pelos contrafortes da Serra do Espinhaço com altitude variando entre 630 e 808m (NUNES \& Pedralli 1995, Pedralli \& Teixeira 1997).

O Clima é do tipo Cwa (Köppen), tropical de altitude com verões amenos e estação seca bem definida entre os meses de maio a setembro e mais úmidos entre os meses de outubro a março (ANTUNES 1986). A hidrografia da Estação pertence à bacia do rio Doce, compreendendo o rio Santa Bárbara e parte do reservatório formado pelo seu represamento, além de córregos e áreas úmidas interiores (Nunes \& Pedralli 1995).

A Estação está inserida na zona de transição Mata Atlân-

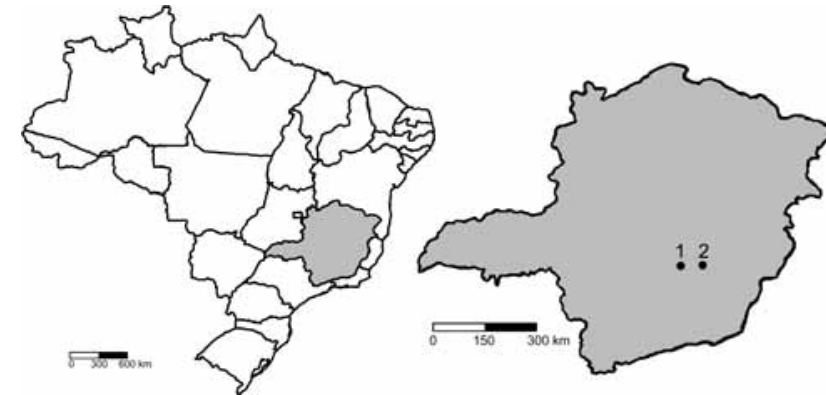

Figura 1. Localização da Estação de Pesquisa e Desenvolvimento Ambiental de Peti no estado de Minas Gerais. (1) Belo Horizonte, (2) EPDA-Peti.

tica e Cerrado, apresentando fisionomias de uma Floresta Estacional Semidecidual (Floresta Mesófila) com manchas de cerrado e vegetação rupícola (Nunes \& Pedralli 1995).

A EPDA-Peti é composta por um mosaico de formações florestais secundárias em diferentes estágios de regeneração. Antes da implantação da usina a área era explorada por atividades de carvoaria. Os diferentes estágios de regeneração da estação se devem ao corte de madeira feitos em diferentes épocas. Após o corte essas áreas foram protegidas contra atividades antrópicas, o que propiciou um processo de regeneração natural (Nunes \& Pedralli 1995).

\section{Métodos}

O levantamento da avifauna da EPDA-Peti foi realizado mensalmente entre abril de 2002 e novembro de 2004. Para o registro das espécies foram utilizados dois métodos principais, a captura e anilhamento com o uso de 'redes de neblina' e o registro áudio-visual em pontos de escuta (BıBBy et al. 1993). Além disso, foram realizadas visitas não sistemáticas aos diferentes ambientes da estação, com o objetivo de se maximizar o levantamento de espécies.

A captura em 'redes de neblina' foi feita em cinco áreas da reserva: Trilha da Cutia (1953"22'S e 4322"21'W), Barragem (1953" 31'Se 4322"07'W), Bonserá (1953"14'Se 43²2" 45'W), Emboque (1953"27'S e 4322" 44'W) e Pomar (1952"41'S e $\left.43^{\circ} 22^{\prime \prime} 08^{\prime} \mathrm{W}\right)$. O esforço total foi de 3480 horas-rede.

A captura foi feita em duas etapas. A primeira delas foi realizada entre julho e novembro de 2002 totalizando 750 horas/rede (150 horas/rede/mês em cada área por período de amostragem). Foram utilizadas 10 redes de neblina (altura de 2,5 m, comprimento de $12 \mathrm{~m}$ e mal ha de $32 \mathrm{~mm}$ ) espaçadas de $10 \mathrm{~m}$ uma das outras. Nessa etapa foram amostradas as Trilhas da Cutia, da Barragem, do Bonserá e do Emboque.

A segunda etapa foi realizada entre fevereiro e agosto de 2004 total izando 2520 horas/rede (120 horas/rede/mês em cada área por período de amostragem). Foram amostradas três áreas: Barragem, Pomar e Bonserá. Nessa etapa foram colocadas 20 redes distribuídas em quatro parcelas fixas de $800 \mathrm{~m}^{2}$ com linhas de cinco redes cada. Em cada uma dessas áreas foram

Revista Brasileira de Zoologia 23 (4): 1217-1230, dezembro 2006 
marcadas quatro parcelas fixas de $800 \mathrm{~m}^{2}$, com uma distância mínima de $100 \mathrm{~m}$. Nessas parcelas foram montadas cinco redes de neblina dispostas em linha.

As aves capturadas foram identificadas no nível de espé cie, com auxílio de guias e chaves de identificação (SCHAUENSEE 1970, Camargo 1986, Ridgely \& Tudor 1989, 1994, Sick 1997, SouzA 2002) e marcados com anilhas metálicas numeradas fornecidas pelo CEMAVE (Centro de Pesquisas para Conservação das Aves Silvestres - IBAmA 1994).

O registro áudio-visual em pontos de escuta foi realizado em três etapas. Para tanto, 35 pontos foram demarcados em sete trilhas principais da estação, espaçados a cada 200 m e a no mínimo $50 \mathrm{~m}$ da borda. Diariamente, sete pontos eram amostrados durante dez minutos cada, em três períodos do dia: madrugada (5:30-7:30 h), meio do dia (11:00-13:00 h) e entardecer (16:0018:00 h). Todos os pontos foram repetidos o mesmo número de vezes e em horários diferentes nos três períodos amostrados. $\mathrm{O}$ tempo máximo de deslocamento entre os pontos era de dez minutos. Para as espécies que vivem em bandos, apenas um registro foi considerado. As três etapas resultaram em esforço amostral de 5250 minutos. As trilhas amostradas foram: Cutia, Macuco (1953'23"Se 04321'58"W), Raposa (1953'30"Se 04321'54"W), Barragem, Trilha Cinco (1953'15"S e 04322'37"W), Trilha Seis (1953'09"S e 4322'41"W) e do Emboque.

Além desses dois métodos principais, foram realizados censos qualitativos em áreas não amostradas pelos métodos anteriores: Mirantes do Cruzeiro (19053"S e 4322"W) e da Pedreira (19-53'04"S e 4322'21"W), Centro de Manejo (19053"S e 43은 22"W) e Alojamento 1 (19-52"14'S e 4322"06'W), Alojamento 2 (1953'06"Se4322'18"W) eAlojamento 3 (1952' 54"S e4322' 05"W), estrada 1 (1953'04"S e 4322'01"W), estrada 2 (1952' 59"S e 4322'26"W) estrada 3 (1953'14"S e 4322'01"W) estrada 4 (1953'18"S e 4321'51"W) Rio Santa Bárbara próximo ao Bom Será (1953'16"S e 4322'28"W) Rio Santa Bárbara na Ponte Pênsil (1952'54"S e 4322'08"W) e reservatório (1953'40"S e 4321'47"W). A amostragem se estendia das 15:30 às 18:30 h e cada ambiente era amostrado por 30 minutos, o que totalizou 1890 minutos de observação. Finalmente, para o registro das aves aquáticas, foram realizadas duas excursões a barco em rotas diversas pela represa.

Para compararmos nossa lista com as espécies levantadas no primeiro inventário, consultamos o relatório técnico não publicado de Carnevalli et al. (1989).

Todas as espécies foram listadas seguindo a taxonomia e a sistemática adotada pelo Comitê Brasileiro de Registros Ornitológicos (CBRo 2005). O status de endemismo da Mata Atlântica segue Stotz et al. (1996) e de endemismo do Cerrado segue Silva \& Bates (2002). O status de conservação segue MACHADo et al. (1998), BirdLifE InTERnAtional (2000) e IbAMA (2003).

Para cada espécie foi designada uma categoria de abundância segundo sua freqüência de ocorrência nos censos feitos em um hábitat específico segundo Rodrigues et al. (2005): A (abundantes): espécies registradas entre 75\% e 100\% das visi- tas a área, isto é, com freqüência de ocorrência entre 75 e 100; C (comuns): com freqüência de ocorrência entre 50 e 74; $E$ (escassas): com freqüência de ocorrência entre 25 e 49; R (raras): com freqüência de ocorrência entre 24 e 2; O (ocasional) com apenas uma observação ao longo de todo o trabalho. As espécies foram classificadas quanto ao seu status migratório como residentes ou migratórias segundo NAKA et al. (2002), Ridgely \& Tudor (1989, 1994), Stotz et al. (1996) e Sick (1997).

\section{RESULTADOS}

\section{Riqueza de espécies}

Foram registradas 231 espécies de aves pertencentes a 57 famílias. A maioria das espécies pertence à família Tyrannidae com 44 espécies, seguidas de Furnariidae com 16 e Thraupidae com 14 espécies (Anexo I).

A primeira etapa de anilhamento resultou em captura de 152 indivíduos pertencentes a 41 espécies. Na segunda etapa foram capturados 371 indivíduos de 54 espécies.

As campanhas utilizando-se o método de pontos de escuta proporcionaram o registro de 128 espécies. Por sua vez, o registro áudio-visual, durante as visitas não sistemáticas a locais diversos, contribuiu com o aumento de outras 70 espécies. Por fim, mais 26 espécies diferentes foram registradas através das excursões a barco.

\section{Composição de espécies}

Vinte e duas espécies foram consi deradas abundantes, 24 comuns, 52 escassas, 90 raras e 43 ocasionais, segundo a freqüência de ocorrência.

A espécie mais freqüentemente capturada foi Pyriglena leucoptera (Vieillot, 1818), com 57 indivíduos, seguidos de Platyrinchus mystaceus Vieillot, 1818 com 27 indivíduos e Basileuterus hypoleucus Bonaparte, 1830 com 21 indivíduos.

Cento e sessenta e duas espécies foram consideradas residentes e provavelmente se reproduzem na região, enquanto 28 são migratórias e 41 não apresentaram nenhum padrão distinto e são consideradas ocasionais na área de estudo (Anexo I).

As espécies Tinamus solitarius (Vieillot, 1819), Penelope obscura Temminck, 1815, Pyroderus scutatus (Shaw, 1792), Sicalis flaveola (Linnaeus, 1766), e Crax blumenbachii Spix, 1825 são consideradas ameaçadas para o estado de Minas Gerais ( $\mathrm{MA}_{\mathrm{A}}$ CHADO et al. 1998), sendo a última também ameaçada globalmente (BIRDLIFE INTERNATIONAL 2000). Ocorrem na estação 34 espécies endêmicas de dois biomas, a Mata Atlântica com 33 representantes e o Cerrado com uma (Anexo I).

Do programa de reintrodução, espécies como T. solitarius, C. blumenbachii, P. superciliaris, Saltator similis, entre outras, são espécies soltas que podem ser visualizadas constantemente. Entretanto, Sporophila lineola (Linnaeus, 1758), S. caerulenscens (Vieillot, 1823), S. albogularis (Spix, 1825), S. angolensis (Linnaeus, 1766), Saltator atricollis Vieillot, 1817, Chrysomus ruficapillus (Vieillot, 1819), Carduel is magellanica (Vieillot, 1805), nunca foram observadas e/ou capturadas. 


\section{Colonização e extinção local}

Em relação ao primeiro inventário realizado por CARNEVALLI et. al. 1989), podem ser apontadas 196 espécies comuns a ambos os inventários (Anexo I). Além disso, 35 espécies novas foram registradas e 52 espécies que estavam na referida lista não foram registradas no presente inventário.

\section{DISCUSSÃO}

\section{Riqueza de espécies}

A Estação de Pesquisa e Desenvolvimento Ambiental de Peti está situada às margens do Rio Santa Bárbara, um dos maiores tributários do Rio Piracicaba que forma o alto da bacia do Rio Doce. O processo de fragmentação da região da bacia do Rio Doce, em Minas Gerais, se iniciou em 1930, período em que a região ainda possuía mais de 50\% de sua cobertura florestal original (FonsECA 1985). Entretanto, a partir de 1930, houve uma enorme devastação da floresta para produção de carvão vegetal, e em 1955, a região encontrava-se total mente fragmentada (FonSECA 1985). M esmo com todo estehistórico de isolamento, podese dizer que a EPDA-Peti ainda abriga uma porção significativa e importante da avifauna da Mata Atlântica, pois nesse local foi registrado um grande número de espécies, cerca de 33\% das 682 espécies já registradas para o bioma da Mata Atlântica (STotz et al. 1996). Este número é proporcionalmente semelhante ao de outras áreas remanescentes e bem conservadas deste bioma, levando-se em conta o tamanho da área (MACHADO \& FonSECA 2000, Anjos 2001). Ribon et al. 2003 encontraram 221 espécies em vários remanescentes florestais na região de Viçosa em Minas Gerais, habitat semelhante morfoclimaticamente à área onde está inserida a EPDA-Peti. Na Serra do Caraça, a cerca de $40 \mathrm{~km}$ da EPDA-Peti foram registradas 287 espécies (Vasconcelos \& MelloJr 2001, Vasconcelos 2003, Vasconcelos et al. 2003a). Essa diferença se deve à pel o menos três fatores, tais como o tamanho da área, o gradiente altitudinal da serra e o melhor estado de conservação de suas matas.

A mata secundária da EPDA-Peti, onde podem ser observados 'talhões' em vários estágios de regeneração, fornece uma heterogeneidade ambiental e sucessional suficiente para garantir que um grande número de espécies florestais possa utilizar e se reproduzir (MóDENA dados não publicados). Isso pode ser dito devido ao el evado número de espécies dependentes de ambientes florestais observados na área, principalmente os pequenos papa-moscas (Tyrannidae) e os papa-formigas (Thamnophilidae) e também devido à presença de espécies consideradas de alta (quatro) e média (vinte) sensibilidade à fragmentação florestal, de acordo com Anjos (2006). Essas espécies não ocorrem em ambientes não florestais, como pastagens e plantações, nem mesmo em florestas de monoculturas como as de Eucalyptus e Pinus, hábitats mais comuns atualmente no sudeste do Brasil. Portanto, as matas da EPDA-Peti, além de suportar um grande número de espécies de aves, abrigam ali espécies florestais, que são aquelas que mais necessitam de esforços conservacionistas, já que as florestas naturais do Brasil estão desaparecendo.
Entretanto, grande parte das espécies florestais observadas ocorre em baixas densidades, e os dados coletados não são suficientes para se afirmar que tais populações estão crescendo ou diminuindo. Algumas espécies foram registradas apenas uma única vez, o que é motivo para estudos mais detalhados na área. Outras espécies, como os grandes jacus frugívoros (Cracidae) ocorrem em grande abundância, e não se sabe qual o impacto destas populações sobre a oferta de frutos na mata, e conseqüentemente, sobre as populações de frugívoros mais especializados, como o próprio pavó, Pyroderus scutatus, ave ameaçada de extinção no estado de Minas Gerais (MACHADO et al. 1998). Estudos sobre as comunidades de frugívoros e dispersores de sementes são essenciais para se estabelecer programas de manejo das aves que dependem deste recurso (HowE 1984, JORDANo et al. 2006).

\section{Composição de espécies}

A área da EPDA-Peti apresenta várias espécies de dois hábitats bastante ameaçados no Brasil, a Mata Atlântica (DEAN 1996) e o Cerrado (OLIVEIRA \& M ARQuis 2002). Isso revela a condição de transição de biomas em que se encontra a área. Uma hipótese alternativa é a de que as aves do Cerrado estariam ampliando suas áreas de distribuição devido ao desmatamento e a presumível aridez local (WILLıs 1991). Nesta opção, as espécies do cerrado seriam possivelmente invasoras na área de estudo, onde estariam expandindo suas áreas de distribuição (veja exemplos em Alvarenga 1990, Vasconcelos 2000, Vasconcelos et al. 2002, Rodrigues \& Gomes 2004, Vasconcelos et al. 2003b). Esse parece ser o caso de Basileuterus flaveolus (Baird, 1865) que não havia sido registrado no inventário anterior. B. flaveolus é uma espécie que ocorre nas matas secas do Cerrado, mas que ultimamente vem sendo registrada em várias áreas de Mata Atlântica (Christiansen \& Piter 1997). Há também o registro de Netta erythrophthalma (Wied, 1832), espécie que vem ampliando sua área de distribuição (WiLlis 1991, Rodrigues \& Michelin 2005).

\section{Colonização e extinção local}

Muitas espécies encontradas no inventário de 1989 não foram registradas no presente trabalho. Algumas delas, entretanto, são espécies oriundas do programa de soltura, que provavelmente não se estabeleceram na área, como por exemplo, o cardeal-do-nordeste Paroaria dominicana (Linnaeus, 1758). Outras são espécies de ocorrência ocasional, como é o caso do beija-flor-de-gravata Augastes scutatus (Temminck, 1824), endêmico de campos rupestres. Esta espécie foi observada uma única vez por CARNEVALLI et al. (1989), e provavelmente tratavase de um indivíduo vagante que se deslocava dos campos rupestre da Serra do Caraça distante a apenas al guns quilômetros da EPDA-Peti. Há espécies, entretanto, que ocorriam naturalmente na área àquela época, e hoje estão localmente extintas. Várias dessas espécies são de ambientes abertos, e provavelmente foram deslocadas para fora da EPDA-Peti à medida que a mata nativa aumentou em extensão e idade, um tipo de extinção previsível e que mostra o quanto a mata estaria rege-

Revista Brasileira de Zoologia 23 (4): 1217-1230, dezembro 2006 
nerando. Há vários exemplos que podem ser colocados dentro deste caso, como Falco sparverius, Taraba major, Anthus lutescens, Sicalis citrina e Ammodramus humeralis. Outras espécies, entretanto, devem ter desaparecido por outros motivos que ainda merecem ser investigados, como, por exemplo, Baryphthengus ruficapillus (Vieillot, 1818).

O presente trabalho registrou 35 espécies que não apareceram no inventário de 1989, o que é um número bastante elevado. Várias podem ser as causas e a natureza dessa diferença. Uma delas, do ponto de vista ecológico, pode estar associada à ocorrência de dois processos opostos. De um lado, a transformação da paisagem da EPDA-Peti, com o crescimento e regeneração da área florestal, de outro, o aumento das áreas desmatadas e/ou antropizadas do entorno da estação. Tais processos, como conseqüência, tornaram a EPDA-Peti um último refúgio florestal para diversas espécies. Resta saber sobre a viabilidade das mesmas em longo prazo.

\section{Manejo de espécies}

Os dados aqui apresentados deverão servir para o delineamento mais preciso do programa de soltura de aves adotado pela direção da EPDA-Peti. Deve-se citar que al gumas espécies soltas nos últimos anos provavelmente não ocorrem naturalmente na área como, por exemplo, Carduelis magellanica (Vieillot, 1805). Assim, algumas destas solturas estariam fadadas ao fracasso, enquanto outras poderiam desestabilizar a comunidade de aves já bastante al terada devido à fragmentação e ao antigo uso da área (monocultura de Eucalyptus).

O canário-da-terra (S. flaveola) é um exemplo de soltura mal sucedida na área, pois dezenas de pássaros dessa espécie foram soltos na EPDA-Peti e até o final desse trabalho nenhum canário-da-terra foi visualizado em toda a área de estudo. A reintrodução de $T$. solitarius, $P$. superciliaris e $C$. blumenbachii tem sido realizada com sucesso, ao menos aparentemente, já que as duas últimas espécies já foram vistas com filhotes. Entretanto, deve-se fazer um estudo para se obter dados mais precisos sobre a readaptação desses animais ao ambiente natural, e mesmo sobre a depen dência da suplementação alimentar nos locais de ceva. Como essas espécies são frugívoras e de grande porte, é importante saber qual o impacto de um aumento populacional dessas espécies sobre outras espécies frugívoras de pequeno porte, como Chiroxiphia caudata (Shaw \& Nodder, 1793) e outros piprídeos e traupídeos dependentes de frutos de sub-bosque. Além disso, é importante que seja feito um acompanhamento dessas espécies para se saber até quando elas devem ser reintroduzidas.

\section{AGRADECIMENTOS}

Ao pessoal da Estação de Pesquisa e Desenvolvimento Ambiental de Peti, principalmente Leotacílio da Fonseca, Maria Beatriz Ferreira, Margareth dos Santos e Maria Elisa Pafilo. Ao Jefferson R. da Silva (Cemig), F. Silveira e A. Kumagai. Ao Centro de Pesquisa para Conservação das Aves Silvestres (CEMAVE-IBAMA) pela concessão das anilhas e licença para anilhamento de aves silvestres; à FUNDEP-UFMG (processo 4870). M.R. agradece ao CNPq (processo 473428/2004-0).

\section{REFERÊNCIAS}

Alvarenga, H.M.F. 1990. Novos registros e expansões geográficas de aves no Leste do Estado de São Paulo. Ararajuba, Rio de Janeiro 1 (1): 115-117.

Anjos, L. Dos. 2001. Bird communities in five Atlantic Forest fragments in southern Brazil. Biotropica, Lawrence, 12: 1127.

ANJos, L. Dos. 2006. Bird specis sensitivity in a fragmented landscape of Atlantic Forest in southern Brazil. Biotropica, Lawrence, 38 (2): 229-234.

Antunes, F.N. 1986. Caracterização climática do Estado de Minas Gerais. Informe Agropecuário, Brasília, 12: 9-13.

Bibby, C.J.; N.J. Collar; M.J. Crosby; F. Heath; C. Imboden; T.H. Johnson; A.J. Long; A.J. Stattersfield \& S.J. Thirgood. 1992. Putting biodiversity on the map: priority areas for global conservation. Cambridge, I.C.B.P, 235p.

BıbBY, C.J.; N.D. Burgess \& D.A. HıLl. 1993. Bird census techniques. London, Academic Press, 257p.

BIRDLIFE INTERnATIONAL. 2000. Threatened birds of the world. Cambridge, Lynx Editions, BirdLife International, 852p.

Brown, K.S. 1987. Conclusions, synthesis, and alternative hypotheses, p. 175-196. In: T.C. Whitmore \& G.T. Prance (Eds). Biogeography and quaternary history in Tropical America. Oxford, Claredon Press, 540p.

CAmARGo, H.F.A. 1986. Contribuição ao estudo das espécies brasileiras do gênero Elaenia (Aves, Tyrannidae). Boletim do Centro de Estudos Ornitológicos, São Paulo, 2 (1): 1-38.

Carnevalli N.; R.B. Machado; A.Brandt; I.R. Lamas; L.V. Lins; L.P. BARROS \& A.L. T SOUZA. 1989. Estudo qualitativo da avifauna da Estação de Pesquisa e Desenvolvimento Ambiental de Peti - EPDA-PETI. Belo Horizonte, UFM G-CEMIG, 65p.

Cbro (Comitê Brasileiro de Registros Ornitológicos). 2005. Lista de aves do Brasil. Disponível na World WideWeb em: http:/ /www.ib.usp.br/cbro [acessado em 15.II.2005].

Christiansen M.B. \& E. Pitter. 1997. Species loss in a forest bird community near Lagoa Santa in southeastern Brazil. Biological Conservation, London, 80: 23-32.

DEAN, W. 1996. A ferro e fogo - a história e a devastação da Mata Atlântica Brasileira. São Paulo, Companhia das Letras, 484p.

FonseCA, G.A.B. 1985. The vanishing Brazilian Atlantic Forest. Biological Conservation, London, 34 (1): 17-34.

Haddad, C.H.F \& I. Sazima. 1992. Anfíbios anuros da Serra do Japi, p. 181-211. In: L.P.C. MorelatTo (Ed.). História Natural da Serra do Japi. Campinas, Editora da Universidade de Campinas, 321p.

Howe, H.F. 1984. Implications of seed dispersal by animals for tropical reserves manegement. Biological Conservation, London, 30: 261-281.

I bama. 1994. Manual de Anilhamento de Aves Silvestres.

Revista Brasileira de Zoologia 23 (4): 1217-1230, dezembro 2006 
Brasília, Instituto Brasileiro do M eio Ambiente e dos Recursos Naturais Renováveis, 146p.

I вама. 2003. Lista das espécies de fauna ameaçada de extinção. Disponíevel na World Wide Web em: http:// www.IBAM A.gov.br/fauna/downloads/lista\%20spp.pdf [acessado em 20.II.2005].

Jordano, P.; M. Galetti; M.A. Piso \& W.R. Silva. 2006. Ligando frugivoria e dispersão de sementes à Biologia da Conservação, p. 411-436. In: C.F.D. Rocha; H.G. Bergallo; M.A.S. Alves; M. VAN SLUYS (Eds). Biologia da Conservação: essências. São Carlos, Rima Editora, 588p.

KINSEY, W.G. 1982. Distribution of primates and forest refuges, p. 455-482. In: G. T. Prance (Ed.). Biological diversification in the tropics. New York, Columbia University Press, 540p.

MachADo, R.B. \& G.A.B. FonseCA. 2000. The avifauna of Rio Doce Valley, southeastern Brazil, a highly fragmented area. Biotropica, Lawrence, 32 (4b): 914-924.

Machado, A.B.M.; G.A.B. FonseCA; R.B. Machado; L.M.S. Aguiar \& L.V. Lins. 1998. Livro vermelho das espécies ameaçadas de extinção da fauna de Minas Gerais. Belo Horizonte, Fundação Biodiversitas, 605p.

Mori S.A.; B.M. Boom; A.M. Carvalho \& T.S. Santos. 1983. Southern Bahian moist forest. Botanical Review, Cambridge, 49 (1): 155-232.

Naka, L.N.; M. Rodrigues; A.L. Roos \& M.A. Azevedo. 2002. Bird conservation on the island of Santa Catarina. Bird Conservation International, Cambridge, 12 (1): 123-150.

Nunes, Y.R.F. \& G. Pedrallı. 1995. Desenvolvimento de metodologia para adensamento e recomposição da vegetação na EPDA-Peti, MG. BIOS, Cadernos do Departamento de Ciências Biológicas da PUC-MG, Belo Horizonte, 2 (1): 5351.

Oliveira, P.S. \& Marquis, R.J. 2002. The Cerrados of Brazil: Ecology and Natural History of a Neotropical Savanna. New York, Columbia University Press, 424p.

Pedralli, G. \& M.C.B. Teixeira 1997. Levantamento florístico e principais fisionomias na Estação de Pesquisa e Desenvolvimento Ambiental de Peti, Santa Bárbara, Estado de Minas Gerais, Brasil. Iheringia, Série Botânica, Porto Alegre, 48 (1): 15-40.

Ribon, R.; J.E. Simon \& G.T. Mattos. 2003. Bird extinction in Atlantic forest fragments of the Viçosa region, Southeastern Brazil. Conservation Biology, Tucson, 17 (6): 1827-1839.

Ridgely, R.S. \& G. TudoR. 1989. The birds of South America. Oxford, University Press, vol. 1, 516p.

Ridgely, R.S. \& G. Tudor. 1994. The birds of South America. Oxford, University Press, vol 2, 814p.

Rodrigues, M. \& H.B. Gomes. 2004. Range extension of the grayheaded tanager Eucometis penicillata (Fringillidae, Emberezinae) in southeastern Brazil. Bulletin of the British
Ornithologists' Club, Londres, 124 (3): 177-184.

Rodrigues, M. \& V.B. Michelin. 2005. Riqueza e diversidade de aves aquáticas de uma lagoa natural no sudeste do Brasil. Revista Brasileira de Zoologia, Curitiba, 22 (4): 928-935.

Rodrigues, M.; L.A. Carrara; L.P. Faria \& H.B. Gomes. 2005. Aves do Parque Nacional da Serra do Cipó: o Vale do Rio Cipó, Minas Gerais, Brasil. Revista Brasileira de Zoologia, Curitiba, 22 (1): 326-338.

Schauensee, R.M. 1970. A guide to the birds of South America. Philadel phia, John Wiley and Sons, 498p.

Sick, H. 1997. Ornitologia brasileira. Rio de Janeiro, Nova fronteira, 912p.

Silva, J.M.C. \& J.M. Bates. 2002. Biogeographic Patterns and Conservation in the South American Cerrado: A Tropical Savanna hotspot. BioScience, Washington, 52 (3): 225-223.

SouzA, D. 2002. All the birds of Brazil: An identification guide. Feira de Santana, Dall, 356p.

Stotz, D.F., J.W. FitzPATRICK, T.A. PARKER III \& D.K. M OSKovits. 1996. Neotropical birds: ecology and conservation. Chicago, The University of Chicago Press, 478p.

Vasconcelos, M.F. 2000. Ocorrência simpátrica de Emberizoides herbicola, Embernagra platensis e Embernagra Iongicauda (Passeriformes: Emberizidae) na região da Serra do Caraça, Minas Gerais. Melopsittacus, Belo Horizonte, 3 (1): 3-5.

VAsconcelos, M.F. 2003. Adições à avifauna da Serra do Caraça, Minas Gerais. Atualidades Ornitológicas, Ivaiporã, 104: 34.

Vasconcelos, M.F. \& T.A. Melo Júnior. 2001. An ornithological survey of Serra do Caraça, Minas Gerais, Brazil. Cotinga, Sandy, 15 (1): 21-31.

Vasconcelos, M.F.; S. D’Angelo-Neto \& M. Rodrigues. 2002. A range extension for the Cipó Canastero Asthenes luizae and the consequences for its conservation status. Bulletin of the British Ornithologists' Club, Londres, 122 (1): 7-10.

Vasconcelos, M.F.; P.N. Vasconcelos; G.N. Maurício; C.A.R. Matrangolo; C.M. Dell'Amore; A. Nemésio; J.C. Ferreira \& E. ENDRIGo. 2003a. Novos registros ornitológicos para a Serra do Caraça, Brasil, com comentários sobre distribuição geográfica de algumas espécies. Lundiana, Belo Horizonte, 4 (2): 135-139.

Vasconcelos, M.F.; M. Maldonado-Coelho \& D.R.C. Buzzetti. 2003b. Range extensions for the Grey-backed Tachuri (Polystictus superciliaris) and the Pale-throated Serra-finch (Embernagra longicauda) with a revision of their geographic distribution. Ornitología Neotropical, St. Louis, 14 (4): 477 489.

WiLlis, E.O. 1991. Expansão geográfica de N etta erythrophtalma, Fluvicola nengeta e outras aves de zonas abertas com a 'desertificação' antrópica em São Paulo. Ararajuba, Rio de Janeiro, 2 (1): 101-102.

Recebido em 27.IV.2006; aceito em 17.XI.2006.

Revista Brasileira de Zoologia 23 (4): 1217-1230, dezembro 2006 
Anexo I. Lista das espécies registradas na EPDA-Peti. A letra C após o nome significa que a espécie foi registrada apenas por CARNEvam et al. (1989) e a letra R espécies observadas apenas no presente estudo; o sinal * representa espécies introduzidas e \# representa espécies introduzidas, mas não registradas no presente trabalho; AM (espécie ameaçada de extinção). Abundância: A (abundantes) espécies com freqüência de ocorrência entre 75 e 100; C (comuns) com freqüência de ocorrência entre 50 e 74; $E$ (escassas) com freqüência de ocorrência entre 25 e 49; R (raras) com freqüência de ocorrência entre 24 e 2; O (ocasional) com apenas uma observação ao longo de todo o trabalho; I (indeterminado). Status migratório: M (migratório), R (residente), I (indeterminado); Endemismo: ATL (Mata Atlântica), CSA (Cerrado); C (Status de conservação): A (ameaçado).

\begin{tabular}{|c|c|c|c|c|}
\hline Nome do táxon & Nome popular & Abundância & Status migratório & Endemismo \\
\hline \multicolumn{5}{|l|}{ Tinamidae } \\
\hline Tinamus solitarius (Vieillot, 1819) * AM & macuco & $\mathrm{E}$ & $\mathrm{R}$ & ATL \\
\hline Crypturellus obsoletus (Temminck, 1815) & inhambuguaçu & $\mathrm{C}$ & $\mathrm{R}$ & \\
\hline Crypturellus parvirostris (Wagler, 1827) & inhambu-chororó & $\mathrm{R}$ & $\mathrm{R}$ & \\
\hline Crypturellus tataupa (Temminck, 1815) R & inhambu-chintã & $\mathrm{O}$ & $\mathrm{R}$ & \\
\hline \multicolumn{5}{|l|}{ Anatidae } \\
\hline Dendrocygna viduata (Linnaeus, 1766) & irerê & $\mathrm{E}$ & M & \\
\hline Dendrocygna autumnalis (Linnaeus, 1758) * & asa-branca & $\mathrm{O}$ & M & \\
\hline Cairina moschata (Linnaeus, 1758)* & pato-do-mato & $\mathrm{E}$ & 1 & \\
\hline Amazonetta brasiliensis (Gmelin, 1789) * & pé-vermelho & $E$ & M & \\
\hline Netta erythrophthalma (Wied, 1832) R & paturi-preta & $\mathrm{O}$ & 1 & \\
\hline \multicolumn{5}{|l|}{ Cracidae } \\
\hline Penelope superciliaris Temminck, 1815 & jacupemba & $\mathrm{C}$ & $\mathrm{R}$ & \\
\hline Penelope obscura Temminck, 1815 AM & jacuaçu & $\mathrm{E}$ & $\mathrm{R}$ & \\
\hline Crax blumenbachii Spix, $1825 *$ AM & mutum-de-bico-vermelho & $\mathrm{C}$ & $\mathrm{R}$ & ATL \\
\hline \multicolumn{5}{|l|}{ Podicipedidae } \\
\hline Tachybaptus dominicus (Linnaeus, 1766) C & merguhão-pequeno & 1 & & \\
\hline Podilymbus podiceps (Linnaeus, 1758) & mergulhão-caçador & $\mathrm{O}$ & M & \\
\hline \multicolumn{5}{|l|}{ Phalacrocoracidae } \\
\hline Phalacrocorax brasilianus (Gmelin, 1789) & biguá & $\mathrm{R}$ & M & \\
\hline \multicolumn{5}{|l|}{ Anhingidae } \\
\hline Anhinga anhinga (Linnaeus, 1766) & biguatinga & $\mathrm{R}$ & 1 & \\
\hline \multicolumn{5}{|l|}{ Ardeidae } \\
\hline Nycticorax nycticorax (Linnaeus, 1758) R & savacu & $\mathrm{R}$ & M & \\
\hline Butorides striata (Linnaeus, 1758) & socozinho & $\mathrm{R}$ & $\mathrm{R}$ & \\
\hline Bubulcus ibis (Linnaeus, 1758) R & garça-vaqueira & $\mathrm{R}$ & $\mathrm{I}$ & \\
\hline Ardea cocoi Linnaeus, 1766 R & garça-moura & $\mathrm{O}$ & 1 & \\
\hline Ardea alba Linnaeus, 1758 & garça-branca-grande & $\mathrm{R}$ & I & \\
\hline Syrigma sibilatrix (Temminck, 1824) R & maria-faceira & $\mathrm{O}$ & I & \\
\hline Egretta thula (Molina, 1782) & garça-branca-pequena & $\mathrm{O}$ & 1 & \\
\hline \multicolumn{5}{|l|}{ Cathartidae } \\
\hline Cathartes aura (Linnaeus, 1758) & urubu-de-cabeça-vermelha & $E$ & $\mathrm{R}$ & \\
\hline Cathartes burrovianus Cassin, 1845) R & urubu-de-cabeça-amarela & $\mathrm{R}$ & $\mathrm{R}$ & ATL \\
\hline Coragyps atratus (Bechstein, 1793) & urubu-de-cabeça-preta & $\mathrm{C}$ & $\mathrm{R}$ & \\
\hline Sarcoramphus papa (Linnaeus, 1758) C & urubu-rei & 1 & & \\
\hline \multicolumn{5}{|l|}{ Pandionidae } \\
\hline Pandion haliaetus (Linnaeus, 1758) C & águia-pescadora & 1 & & \\
\hline \multicolumn{5}{|l|}{ Accipitridae } \\
\hline Leptodon cayanensis (Latham, 1790) C & gavião-de-cabeça-cinza & 1 & & \\
\hline Geranospiza caerulescens (Vieillot, 1817) C & gavião-pernilongo & 1 & & \\
\hline Rupornis magnirostris (Gmelin, 1788) & gavião-carijó & $A$ & $\mathrm{R}$ & \\
\hline Buteo albicaudatus Vieillot, 1816 C & gavião-de-rabo-branco & 1 & & \\
\hline
\end{tabular}


Anexo I. Continuação.

\begin{tabular}{|c|c|c|c|c|}
\hline Nome do táxon & Nome popular & Abundância & Status migratório & Endemismo \\
\hline \multicolumn{5}{|l|}{ Falconidae } \\
\hline Caracara plancus (Miller, 1777) & caracará & E & $\mathrm{R}$ & \\
\hline Herpetotheres cachinnans (Linnaeus, 1758) & acauã & $\mathrm{R}$ & 1 & \\
\hline Milvago chimachima (Vieillot, 1816) & carrapateiro & A & $\mathrm{R}$ & \\
\hline Micrastur semitorquatus (Vieillot, 1817) R & falcão-relógio & $\mathrm{R}$ & $\mathrm{R}$ & \\
\hline Falco sparverius Linnaeus, 1758 C & quiriquiri & 1 & & \\
\hline Falco rufigularis Daudin, 1800 & cauré & $\mathrm{O}$ & 1 & \\
\hline \multicolumn{5}{|l|}{ Rallidae } \\
\hline Aramides saracura (Spix, 1825) & saracura-do-mato & $\mathrm{R}$ & $\mathrm{R}$ & \\
\hline Laterallus melanophaius (Vieillot, 1819) C & sanã-parda & I & & \\
\hline Porzana albicollis (Vieillot, 1819) C & sanã-carijó & I & & \\
\hline Pardirallus nigricans (Vieillot, 1819) R & saracura-sanã & $\mathrm{R}$ & $\mathrm{R}$ & \\
\hline Gallinula chloropus (Linnaeus, 1758) & frango-d'água-comum & $\mathrm{O}$ & $\mathrm{R}$ & \\
\hline Porphyrio martinica (Linnaeus, 1766) R & frango-d'água-azul & $\mathrm{O}$ & $\mathrm{R}$ & \\
\hline \multicolumn{5}{|l|}{ Cariamidae } \\
\hline Cariama cristata (Linnaeus, 1766) & seriema & $\mathrm{O}$ & 1 & \\
\hline \multicolumn{5}{|l|}{ Jacanidae } \\
\hline Jacana jacana (Linnaeus, 1766) & jaçanã & $\mathrm{R}$ & $\mathrm{R}$ & \\
\hline \multicolumn{5}{|l|}{ Recurvirostrudae } \\
\hline Himantopus melanurus Vieillot, 1817 R & pernilongo-de-costas-brancas & 1 & M & \\
\hline \multicolumn{5}{|l|}{ Charadriidae } \\
\hline Vanellus cayanus (Latham, 1790) C & batuíra-de-esporão & 1 & & \\
\hline Vanellus chilensis (Molina, 1782) & quero-quero & E & $\mathrm{R}$ & \\
\hline \multicolumn{5}{|l|}{ Scolopacidae } \\
\hline Tringa melanoleuca (Gmelin, 1789) R & maçarico-grande-de-perna-amarela & $\mathrm{R}$ & M & \\
\hline Tringa flavipes (Gmelin, 1789) C & maçarico-de-perna-amarela & I & & \\
\hline Actitis macularius (Linnaeus, 1766) C & maçarico-pintado & I & & \\
\hline \multicolumn{5}{|l|}{ Sternidae } \\
\hline Sterna hirundo Linnaeus, 1758 C & trinta-réis-boreal & 1 & & \\
\hline Sternula superciliaris (Vieillot, 1819) C & trinta-réis-anão & I & & \\
\hline \multicolumn{5}{|l|}{ Columbidae } \\
\hline Columbina talpacoti (Temminck, 1811)* & rolinha-roxa & E & $\mathrm{R}$ & \\
\hline Columbina squammata (Lesson, 1831) & fogo-apagou & E & $\mathrm{R}$ & \\
\hline Columbina picui (Temminck, 1813) & rolinha-picui & $\mathrm{R}$ & $\mathrm{R}$ & \\
\hline Claravis pretiosa (Ferrari-Perez, 1886) & pararu-azul & $\mathrm{R}$ & 1 & \\
\hline Patagioenas picazuro (Temminck, 1813)* & pombão & E & $\mathrm{R}$ & \\
\hline Patagioenas plumbea (Vieillot, 1818) & pomba-amargosa & C & $\mathrm{R}$ & \\
\hline Leptotila verreauxi Bonaparte, 1855 & juriti-pupu & E & $\mathrm{R}$ & \\
\hline Leptotila rufaxilla (Richard \& Bernard, 1792) & juriti-gemedeira & E & $\mathrm{R}$ & \\
\hline Geotrygon montana (Linnaeus, 1758) & pariri & $\mathrm{R}$ & I & \\
\hline \multicolumn{5}{|l|}{ Psittacidae } \\
\hline Aratinga leucophthalma (Statius Muller, 1776) * & periquitão-maracanã & E & $\mathrm{R}$ & \\
\hline Forpus xanthopterygius (Spix, 1824) & tuim & $\mathrm{E}$ & $\mathrm{R}$ & \\
\hline Brotogeris chiriri (Vieillot, 1818) R* & periquito-de-encontro-amarelo & $\mathrm{E}$ & $\mathrm{R}$ & \\
\hline Pionus maximiliani (Kuhl, 1820) & maitaca-verde & $\mathrm{O}$ & $\mathrm{R}$ & \\
\hline
\end{tabular}

Revista Brasileira de Zoologia 23 (4): 1217-1230, dezembro 2006 
Anexo I. Continuação.

\begin{tabular}{|c|c|c|c|c|}
\hline Nome do táxon & Nome popular & Abundância & a Status migratório & Endemismo \\
\hline \multicolumn{5}{|l|}{ Cuculidae } \\
\hline Piaya cayana (Linnaeus, 1766) & alma-de-gato & $\mathrm{E}$ & $\mathrm{R}$ & \\
\hline Crotophaga ani Linnaeus, 1758 & anu-preto & $\mathrm{R}$ & $\mathrm{R}$ & \\
\hline Guira guira (Gmelin, 1788) & anu-branco & $\mathrm{R}$ & $\mathrm{R}$ & \\
\hline \multicolumn{5}{|l|}{ Tytonidae } \\
\hline Tyto alba (Scopoli, 1769) & coruja-da-igreja & $\mathrm{R}$ & $\mathrm{R}$ & \\
\hline \multicolumn{5}{|l|}{ Strigidae } \\
\hline M egascops choliba (Vieillot, 1817) & corujinha-do-mato & $E$ & $\mathrm{R}$ & \\
\hline Pulsatrix perspicillata (Latham, 1790) R & murucututu & $\mathrm{R}$ & $\mathrm{R}$ & \\
\hline Strix virgata (Cassin, 1849) & coruja-do-mato & $\mathrm{R}$ & $\mathrm{R}$ & \\
\hline Glaucidium brasilianum (Gmelin, 1788) C & caburé & I & & \\
\hline Athene cunicularia (Molina, 1782) R & coruja-buraqueira & $\mathrm{O}$ & $\mathrm{R}$ & \\
\hline Asio stygius (Wagler, 1832) R & mocho-diabo & $\mathrm{R}$ & $\mathrm{R}$ & \\
\hline \multicolumn{5}{|l|}{ Nyctibiidae } \\
\hline Nyctibius griseus (Gmelin, 1789) & mãe-da-lua & $\mathrm{R}$ & $\mathrm{R}$ & \\
\hline \multicolumn{5}{|l|}{ Caprimulgidae } \\
\hline Lurocalis semitorquatus (Gmelin, 1789) & tuju & $\mathrm{R}$ & $\mathrm{R}$ & \\
\hline Nyctidromus albicollis (Gmelin, 1789) & bacurau & $\mathrm{C}$ & $\mathrm{R}$ & \\
\hline Nyctiphrynus ocellatus (Tschudi, 1844) C & bacurau-ocelado & 1 & & \\
\hline Caprimulgus rufus Boddaert, 1783 & joão-corta-pau & $\mathrm{O}$ & 1 & \\
\hline Caprimulgus longirostris Bonaparte, $1825 \mathrm{C}$ & bacurau-da-telha & I & & \\
\hline Hydropsalis torquata (Gmelin, 1789) & bacurau-tesoura & $\mathrm{E}$ & $\mathrm{R}$ & \\
\hline \multicolumn{5}{|l|}{ Apodidae } \\
\hline Streptoprocne zonaris (Shaw, 1796) & taperuçu-de-coleira-branca & $\mathrm{E}$ & M & \\
\hline Chaetura cinereiventris Sclater, 1862 C & andorinhão-de-sobre-cinzento & 1 & & \\
\hline Chaetura meridionalis Hellmayr, 1907 C & andorinhão-do-temporal & 1 & & \\
\hline \multicolumn{5}{|l|}{ Trochilidae } \\
\hline Glaucis hirsutus (Gmelin, 1788) C & balança-rabo-de-bico-torto & $\mathrm{I}$ & & \\
\hline Phaethornis squalidus (Temminck, 1822) & rabo-branco-pequeno & $\mathrm{R}$ & $\mathrm{R}$ & \\
\hline Phaethornis pretrei (Lesson \& Delattre, 1839) & rabo-branco-acanelado & $\mathrm{E}$ & $\mathrm{R}$ & \\
\hline Eupetomena macroura (Gmelin, 1788) & beija-flor-tesoura & $E$ & $\mathrm{R}$ & \\
\hline Aphantochroa cirrochloris (Vieillot, 1818) R & beija-flor-cinza & $\mathrm{R}$ & I & ATL \\
\hline Florisuga fusca (Vieillot, 1817) & beija-flor-preto & $\mathrm{R}$ & M & ATL \\
\hline $\begin{array}{l}\text { Chlorostilbon aureoventris (d'Orbigny \& Lafresnaye, } \\
\text { 1838) }\end{array}$ & besourinho-de-bico-vemelho & $\mathrm{R}$ & $\mathrm{R}$ & \\
\hline Thalurania furcata (Gmelin, 1788) R & beija-flor-tesoura-verde & $\mathrm{E}$ & $\mathrm{R}$ & \\
\hline Thalurania glaucopis (Gmelin, 1788) & beija-flor-de-fronte-violeta & $\mathrm{R}$ & $\mathrm{R}$ & ATL \\
\hline Hylocharis cyanus (Vieillot, 1818) & beija-flor-roxo & $\mathrm{R}$ & $\mathrm{I}$ & \\
\hline Amazilia lactea (Lesson, 1832) & beija-flor-de-peito-azul & $\mathrm{C}$ & $\mathrm{R}$ & \\
\hline \multicolumn{5}{|l|}{ Trogonidae } \\
\hline Trogon surrucura Vieillot, 1817 & surucuá-variado & $\mathrm{C}$ & $\mathrm{R}$ & ATL \\
\hline \multicolumn{5}{|l|}{ Alcedinidae } \\
\hline Ceryle torquatus (Linnaeus, 1766) & martim-pescador-grande & $\mathrm{E}$ & $\mathrm{R}$ & \\
\hline Chloroceryle amazona (Latham, 1790) & martim-pescador-verde & $\mathrm{R}$ & $\mathrm{R}$ & \\
\hline Chloroceryle americana (Gmelin, 1788) & martim-pescador-pequeno & $\mathrm{R}$ & $\mathrm{R}$ & \\
\hline
\end{tabular}


Anexo I. Continuação.

\begin{tabular}{|c|c|c|c|c|}
\hline Nome do táxon & Nome popular & Abundância & Status migratório & Endemismo \\
\hline \multicolumn{5}{|l|}{ Momotidae } \\
\hline Baryphthengus ruficapillus (Vieillot, 1818) C & juruva-verde & I & & \\
\hline \multicolumn{5}{|l|}{ Galbulidae } \\
\hline Galbula ruficauda Cuvier, 1816 & ariramba-de-cauda-ruiva & $\mathrm{R}$ & $\mathrm{R}$ & \\
\hline \multicolumn{5}{|l|}{ Bucconidae } \\
\hline Malacoptila striata (Spix, 1824) & barbudo-rajado & 0 & $\mathrm{R}$ & ATL \\
\hline Nonnula rubecula (Spix, 1824) C & macuru & I & & \\
\hline \multicolumn{5}{|l|}{ Picidae } \\
\hline Picumnus cirratus Temminck, 1825 & pica-pau-anão-barrado & A & $\mathrm{R}$ & \\
\hline M elanerpes candidus (Otto, 1796) & birro, pica-pau-branco & $\mathrm{R}$ & $\mathrm{R}$ & \\
\hline Veniliornis passerinus (Linnaeus, 1766) & picapauzinho-anão & $\mathrm{E}$ & $\mathrm{R}$ & \\
\hline Piculus chrysochloros (Vieillot, 1818) R & pica-pau-dourado-escuro & $\mathrm{O}$ & $\mathrm{R}$ & \\
\hline Colaptes melanochloros (Gmelin, 1788) & pica-pau-verde-barrado & $\mathrm{R}$ & $\mathrm{R}$ & \\
\hline Colaptes campestris (Vieillot, 1818) & pica-pau-do-campo & $\mathrm{R}$ & $\mathrm{R}$ & \\
\hline Celeus flavescens (Gmelin, 1788) & pica-pau-de-cabeça-amarela & $\mathrm{R}$ & $\mathrm{R}$ & \\
\hline Dryocopus lineatus (Linnaeus, 1766) & pica-pau-de-banda-branca & $\mathrm{R}$ & R & \\
\hline \multicolumn{5}{|l|}{ Thamnophilidae } \\
\hline Mackenziaena leachii (Such, 1825) & borralhara-assobiadora & $\mathrm{R}$ & $\mathrm{R}$ & ATL \\
\hline Mackenziaena severa (Lichtenstein, 1823) C & borralhara & 1 & & \\
\hline Taraba major (Vieillot, 1816) C & chorá-boi & I & & \\
\hline Thamnophilus caerulescens Vieillot, 1816 & choca-da-mata & A & $\mathrm{R}$ & \\
\hline Thamnophilus ruficapillus Vieillot, $1816 \mathrm{R}$ & choca-de-chapéu-vermelho & 0 & $R$ & \\
\hline Dysithamnus mentalis (Temminck, 1823) & choquinha-lisa & $\mathrm{E}$ & $R$ & \\
\hline Herpsilochmus atricapillus Pelzeln, 1868 & chorozinho-de-chapéu-preto & A & $\mathrm{R}$ & \\
\hline Herpsilochmus rufimarginatus (Temminck, 1822) R & chorozinho-de-asa-vermelha & 0 & $\mathrm{R}$ & \\
\hline Formicivora serrana Hellmayr, 1929 & formigueiro-da-serra & $\mathrm{E}$ & $\mathrm{R}$ & ATL \\
\hline Drymophila ochropyga (Hellmayr, 1906) & choquinha-de-dorso-vermelho & C & $\mathrm{R}$ & ATL \\
\hline Pyriglena leucoptera (Vieillot, 1818) & papa-taoca-do-sul & A & $\mathrm{R}$ & ATL \\
\hline Myrmeciza loricata (Lichtenstein, 1823) & formigueiro-assobiador & C & $\mathrm{R}$ & ATL \\
\hline \multicolumn{5}{|l|}{ Conopophagidae } \\
\hline Conopophaga lineata (Wied, 1831) & chupa-dente & E & $\mathrm{R}$ & ATL \\
\hline \multicolumn{5}{|l|}{ Rhinocryptidae } \\
\hline Scytalopus indigoticus (Wied, 1831) R & macuquinho & $\mathrm{R}$ & $\mathrm{R}$ & ATL \\
\hline \multicolumn{5}{|l|}{ Dendrocolaptidae } \\
\hline Sittasomus griseicapillus (Vieillot, 1818) & arapaçu-verde & A & $\mathrm{R}$ & \\
\hline Dendrocolaptes platyrostris Spix, 1825 & arapaçu-grande & $\mathrm{O}$ & $\mathrm{R}$ & \\
\hline Xiphorhynchus fuscus (Vieillot, 1818) & arapaçu-rajado & $\mathrm{R}$ & $\mathrm{R}$ & ATL \\
\hline Lepidocolaptes squamatus (Lichtenstein, 1822) & arapaçu-escamado & $\mathrm{E}$ & $\mathrm{R}$ & ATL \\
\hline \multicolumn{5}{|l|}{ Furnariidae } \\
\hline Furnarius figulus (Lichtenstein, 1823) R & casaca-de-couro-da-lama & $\mathrm{R}$ & $\mathrm{R}$ & \\
\hline Furnarius rufus (Gmelin, 1788) & joão-de-barro & $\mathrm{R}$ & $\mathrm{R}$ & \\
\hline Synallaxis ruficapilla Vieillot, 1819 & pichororé & C & $\mathrm{R}$ & ATL \\
\hline Synallaxis cinerascens Temminck, $1823 \mathrm{R}$ & pi-puí & $\mathrm{R}$ & $\mathrm{R}$ & \\
\hline Synallaxis frontalis Pelzeln, $1859 \mathrm{R}$ & petrim & $\mathrm{O}$ & R & \\
\hline Synallaxis albescens Temminck, 1823 & uí-pi & $\mathrm{R}$ & $\mathrm{R}$ & \\
\hline
\end{tabular}


Anexo I. Continuação.

\begin{tabular}{|c|c|c|c|c|}
\hline Nome do táxon & Nome popular & Abundância & Status migratório & Endemismo \\
\hline Synallaxis spixi Sclater, 1856 & joão-teneném & $\mathrm{E}$ & $\mathrm{R}$ & \\
\hline Synallaxis scutata Sclater, 1859 & estrelinha-preta & 0 & $\mathrm{R}$ & \\
\hline Cranioleuca pallida (Wied, 1831) C & arredio-pálido & I & & \\
\hline Certhiaxis cinnamomeus (Gmelin, 1788) & curutié & $\mathrm{O}$ & 1 & \\
\hline Phacellodomus rufifrons (Wied, 1821) & joão-de-pau & $\mathrm{E}$ & $\mathrm{R}$ & \\
\hline Phacellodomus ruber (Vieillot, 1817) & graveteiro & 0 & I & \\
\hline Phacellodomus erythrophthalmus (Wied, 1821) C & joão-botina-da-mata & 1 & & \\
\hline Syndactyla rufosuperciliata (Lafresnaye, 1832) & trepador-quiete & $\mathrm{R}$ & $\mathrm{R}$ & \\
\hline Philydor rufum (Vieillot, 1818) & limpa-folha-de-testa-baia & $\mathrm{R}$ & $\mathrm{R}$ & \\
\hline Automolus leucophthalmus (Wied, 1821) & barranqueiro-de-olho-branco & $\mathrm{E}$ & $\mathrm{R}$ & ATL \\
\hline Lochmias nematura (Lichtenstein, 1823) & joão-porca & $\mathrm{R}$ & $\mathrm{R}$ & \\
\hline Xenops rutilans Temminck, 1821 & bico-virado-carijó & $\mathrm{R}$ & $\mathrm{R}$ & \\
\hline \multicolumn{5}{|l|}{ Tyrannidae } \\
\hline Mionectes rufiventris Cabanis, 1846 & abre-asa-de-cabeça-cinza & E & $\mathrm{R}$ & ATL \\
\hline Leptopogon amaurocephalus Tschudi, 1846 & cabeçudo & $\mathrm{E}$ & $\mathrm{R}$ & \\
\hline Corythopis delalandi (Lesson, 1830) & estalador & $\mathrm{R}$ & $\mathrm{R}$ & \\
\hline Hemitriccus nidipendulus (Wied, 1831) & tachuri-campainha & $\mathrm{R}$ & $\mathrm{R}$ & ATL \\
\hline Poecilotriccus plumbeiceps (Lafresnaye, 1846) & tororó & C & $\mathrm{R}$ & \\
\hline Todirostrum poliocephalum (Wied, 1831) & teque-teque & $\mathrm{E}$ & $\mathrm{R}$ & ATL \\
\hline Phyllomyias fasciatus (Thunberg, 1822) & piolhinho & $\mathrm{E}$ & $\mathrm{R}$ & \\
\hline Myiopagis caniceps (Swainson, 1835) R & guaracava-cinzenta & $\mathrm{R}$ & $\mathrm{R}$ & \\
\hline Myiopagis viridicata (Vieillot, 1817) & guaracava-de-crista-alaranjada & $\mathrm{R}$ & $\mathrm{R}$ & \\
\hline Elaenia flavogaster (Thunberg, 1822) & guaracava-de-barriga-amarela & $\mathrm{E}$ & $\mathrm{R}$ & \\
\hline Elaenia parvirostris Pelzeln, 1868 & guaracava-de-bico-curto & O & 1 & \\
\hline Elaenia mesoleuca (Deppe, 1830) & tuque & 0 & 1 & \\
\hline Elaenia obscura (d'Orbigny \& Lafresnaye, 1837) & tucão & $\mathrm{R}$ & $\mathrm{R}$ & \\
\hline Camptostoma obsoletum (Temminck, 1824) & risadinha & 0 & 1 & \\
\hline Serpophaga subcristata (Vieillot, 1817) C & alegrinho & 1 & & \\
\hline Phaeomyias murina (Spix, 1825) & bagageiro & $\mathrm{R}$ & $\mathrm{R}$ & \\
\hline Capsiempis flaveola (Lichtenstein, 1823) R & marianinha-amarela & $\mathrm{O}$ & $\mathrm{R}$ & \\
\hline Euscarthmus meloryphus Wied, 1831 C & barulhento & 1 & & \\
\hline Phylloscartes ventralis (Temminck, 1824) & borboletinha-do-mato & 0 & 1 & \\
\hline M yiornis auricularis (Vieillot, 1818) & miudinho & $\mathrm{R}$ & $\mathrm{R}$ & ATL \\
\hline Tolmomyias sulphurescens (Spix, 1825) & bico-chato-de-orelha-preta & A & $\mathrm{R}$ & \\
\hline Platyrinchus mystaceus Vieillot, 1818 & patinho & C & $\mathrm{R}$ & \\
\hline Myiophobus fasciatus (Statius Muller, 1776) & filipe & $\mathrm{R}$ & $\mathrm{R}$ & \\
\hline Myiobius barbatus (Gmelin, 1789) & assanhadinho & $\mathrm{O}$ & $\mathrm{R}$ & \\
\hline M yiophobus fasciatus (Statius Muller, 1776) C & filipe & 1 & & \\
\hline Hirundinea ferruginea (Gmelin, 1788) & gibão-de-couro & $\mathrm{E}$ & M & \\
\hline Lathrotriccus euleri (Cabanis, 1868) & enferrujado & C & $\mathrm{R}$ & \\
\hline Knipolegus lophotes Boie, 1828 & maria-preta-de-penacho & $\mathrm{R}$ & 1 & \\
\hline Knipolegus nigerrimus (Vieillot, 1818) & maria-preta-de-garganta-vermelha & $\mathrm{R}$ & 1 & ATL \\
\hline Satrapa icterophrys (Vieillot, 1818) R & suiriri-pequeno & $\mathrm{R}$ & $\mathrm{R}$ & \\
\hline Xolmis velatus (Lichtenstein, 1823) C & noivinha-branca & 1 & & \\
\hline Gubernetes yetapa (Vieillot, 1818) C & tesoura-do-brejo & 1 & & \\
\hline
\end{tabular}


Anexo I. Continuação.

\begin{tabular}{|c|c|c|c|c|}
\hline Nome do táxon & Nome popular & \multicolumn{3}{|c|}{ Abundância Status migratório Endemismc } \\
\hline M uscipipra vetula (Lichtenstein, 1823) C & tesoura-cinzenta & 1 & \multirow{2}{*}{\multicolumn{2}{|c|}{$\mathrm{R}$}} \\
\hline Fluvicola nengeta (Linnaeus, 1766) & lavadeira-mascarada & $\mathrm{E}$ & & \\
\hline Arundinicola leucocephala (Linnaeus, 1764) C & freirinha & 1 & & \\
\hline Colonia colonus (Vieillot, 1818) & viuvinha & C & \multicolumn{2}{|l|}{$\mathrm{R}$} \\
\hline Machetornis rixosa (Vieillot, 1819) & suiriri-cavaleiro & $\mathrm{O}$ & \multicolumn{2}{|l|}{ I } \\
\hline Legatus leucophaius (Vieillot, 1818) & bem-te-vi-pirata & $\mathrm{R}$ & \multicolumn{2}{|l|}{1} \\
\hline M yiozetetes cayanensis (Linnaeus, 1766) & bentevizinho-de-asa-ferrugínea & $\mathrm{R}$ & \multicolumn{2}{|l|}{$\mathrm{R}$} \\
\hline M yiozetetes similis (Spix, 1825) & bentevizinho-de-penacho-vermelho & A & \multicolumn{2}{|l|}{$\mathrm{R}$} \\
\hline Pitangus sulphuratus (Linnaeus, 1766) & bem-te-vi & A & \multicolumn{2}{|l|}{$\mathrm{R}$} \\
\hline Myiodynastes maculatus (Statius Muller, 1776) & bem-te-vi-rajado & E & \multicolumn{2}{|l|}{ M } \\
\hline Megarynchus pitangua (Linnaeus, 1766) & neinei & A & \multicolumn{2}{|l|}{ M } \\
\hline Tyrannus albogularis Burmeister, 1856 & suiriri-de-garganta-branca & $\mathrm{R}$ & \multicolumn{2}{|l|}{ I } \\
\hline Tyrannus melancholicus Vieillot, 1819 & suirin & $\mathrm{E}$ & \multicolumn{2}{|l|}{ M } \\
\hline Tyrannus savana Vieillot, 1808 & tesourinha & $\mathrm{R}$ & \multicolumn{2}{|l|}{ M } \\
\hline Sirystes sibilator (Vieillot, 1818) & gritador & $\mathrm{R}$ & \multicolumn{2}{|l|}{1} \\
\hline Casiornis rufus (Vieillot, 1816) R & caneleiro & $\mathrm{R}$ & \multicolumn{2}{|l|}{$\mathrm{R}$} \\
\hline Myiarchus swainsoni Cabanis \& Heine, 1859 R & irré & $\mathrm{O}$ & \multicolumn{2}{|l|}{ I } \\
\hline Myiarchus ferox (Gmelin, 1789) & maria-cavaleira & A & \multicolumn{2}{|l|}{ M } \\
\hline M yiarchus tyrannulus (Statius Muller, 1776) R & maria-cavaleira-de-rabo-enferrujado & $\mathrm{R}$ & \multicolumn{2}{|l|}{ M } \\
\hline \multicolumn{5}{|l|}{ Cotingidae } \\
\hline Pyroderus scutatus (Shaw, 1792) AM & pavó & $\mathrm{E}$ & $\mathrm{R}$ & ATL \\
\hline \multicolumn{5}{|l|}{ Pipridae } \\
\hline Neopelma pallescens (Lafresnaye, 1853) R & fruxu-do-cerradão & $\mathrm{R}$ & \multicolumn{2}{|l|}{$\mathrm{R}$} \\
\hline Neopelma aurifrons (Wied, 1831) C & fruxu-baiano & 1 & & \\
\hline Ilicura militaris (Shaw \& Nodder, 1809) & tangarazinho & A & $\mathrm{R}$ & ATL \\
\hline Manacus manacus (Linnaeus, 1766) & rendeira & C & $\mathrm{R}$ & \\
\hline Chiroxiphia caudata (Shaw \& Nodder, 1793) & tangará & A & $\mathrm{R}$ & ATL \\
\hline \multicolumn{5}{|l|}{ Tityridae } \\
\hline Schiffornis virescens (Lafresnaye, 1838) & flautim & E & $\mathrm{R}$ & ATL \\
\hline Pachyramphus polychopterus (Vieillot, 1818) & caneleiro-preto & $\mathrm{R}$ & \multicolumn{2}{|c|}{$\mathrm{R}$} \\
\hline Pachyramphus viridis (Vieillot, 1816) C & caneleiro-verde & 1 & & \\
\hline Pachyramphus castaneus (Vieillot, 1818) C & caneleiro & 1 & & \\
\hline Pachyramphus validus (Lichtenstein, 1823) $R$ & caneleiro-de-chapéu-preto & $\mathrm{R}$ & $\mathrm{R}$ & \\
\hline Vireonidae & & & & \\
\hline Cyclarhis gujanensis (Gmelin, 1789) & pitiguari & A & $\mathrm{R}$ & \\
\hline Vireo olivaceus (Linnaeus, 1766) & juruviara & $\mathrm{R}$ & M & \\
\hline Hylophilus amaurocephalus (Nordmann, 1835) & vite-vite-de-olho-cinza & $\mathrm{R}$ & $\mathrm{R}$ & ATL \\
\hline Corvidae & & & & \\
\hline Cyanocorax cristatellus (Temminck, 1823) C & gralha-do-campo & 1 & & \\
\hline Hirundinidae & & & & \\
\hline Tachycineta albiventer (Boddaert, 1783) & andorinha-do-rio & $\mathrm{R}$ & M & \\
\hline Tachycineta leucorrhoa (Vieillot, 1817) & andorinha-de-sobre-branco & $\mathrm{R}$ & M & \\
\hline Progne tapera (Vieillot, 1817) & andorinha-do-campo & $\mathrm{E}$ & M & \\
\hline Progne chalybea (Gmelin, 1789) C & andorinha-doméstica-grande & I & & \\
\hline Pygochelidon cyanoleuca (Vieillot, 1817) & andorinha-pequena-de-casa & $\mathrm{E}$ & M & \\
\hline Stelgidopteryx ruficollis (Vieillot, 1817) & andorinha-serradora & $\mathrm{R}$ & M & \\
\hline
\end{tabular}


Anexo I. Continuação.

\begin{tabular}{|c|c|c|c|c|}
\hline Nome do táxon & Nome popular & Abundância & Status migratório & Endemismo \\
\hline \multicolumn{5}{|l|}{ Troglodytidae } \\
\hline Troglodytes musculus Naumann, 1823 & corruíra & $\mathrm{C}$ & $\mathrm{R}$ & \\
\hline \multicolumn{5}{|l|}{ Turdidae } \\
\hline Platycichla flavipes (Vieillot, 1818) & sabiá-una & $\mathrm{O}$ & M & \\
\hline Turdus subalaris (Seebohm, 1887) & sabiá-ferreiro & $\mathrm{O}$ & M & \\
\hline Turdus rufiventris Vieillot, $1818 *$ & sabiá-laranjeira & $\mathrm{C}$ & $\mathrm{R}$ & \\
\hline Turdus leucomelas Vieillot, $1818 *$ & sabiá-barranco & A & $\mathrm{R}$ & \\
\hline Turdus amaurochalinus Cabanis, $1850 *$ & sabiá-poca & $\mathrm{R}$ & M & \\
\hline Turdus albicollis Vieillot, 1818 & sabiá-coleira & $\mathrm{R}$ & $\mathrm{R}$ & \\
\hline \multicolumn{5}{|l|}{ Mimidae } \\
\hline M imus saturninus (Lichtenstein, 1823) & sabiá-do-campo & $\mathrm{O}$ & $\mathrm{R}$ & \\
\hline \multicolumn{5}{|l|}{ Motacillidae } \\
\hline Anthus lutescens Pucheran, 1855 C & caminheiro-zumbidor & 1 & & \\
\hline \multicolumn{5}{|l|}{ Coerebidae } \\
\hline Coereba flaveola (Linnaeus, 1758) & cambacica & A & $\mathrm{R}$ & \\
\hline \multicolumn{5}{|l|}{ Thraupidae } \\
\hline Schistochlamys ruficapillus (Vieillot, 1817) * & bico-de-veludo & $\mathrm{R}$ & $\mathrm{R}$ & \\
\hline Cissopis leverianus (Gmelin, 1788) C & tietinga & 1 & & \\
\hline Nemosia pileata (Boddaert, 1783) & saíra-de-chapéu-preto & $\mathrm{R}$ & $\mathrm{R}$ & \\
\hline Thlypopsis sordida (d'Orbigny \& Lafresnaye, 1837) & saí-canário & $\mathrm{R}$ & $\mathrm{R}$ & \\
\hline Trichothraupis melanops (Vieillot, 1818) & tiê-de-topete & $\mathrm{E}$ & $\mathrm{R}$ & \\
\hline Piranga flava (Vieillot, 1822) C & tiê-do-mato-grosso & 1 & & \\
\hline Tachyphonus coronatus (Vieillot, 1822) * & tiê-preto & $\mathrm{E}$ & $\mathrm{R}$ & ATL \\
\hline Thraupis sayaca (Linnaeus, 1766) & sanhaçu-cinzento & $\mathrm{C}$ & $\mathrm{R}$ & \\
\hline Thraupis ornata (Sparrman, 1789)* & sanhaçu-de-encontro-amarelo & $\mathrm{E}$ & $\mathrm{R}$ & ATL \\
\hline Thraupis palmarum (Wied, 1823)* & sanhaçu-do-coqueiro & $\mathrm{C}$ & $\mathrm{R}$ & \\
\hline Tangara cyanoventris (Vieillot, 1819) & saíra-douradinha & $\mathrm{E}$ & $\mathrm{R}$ & ATL \\
\hline Tangara desmaresti (Vieillot, 1819) C & saíra-lagarta & 1 & & \\
\hline Tangara cayana (Linnaeus, 1766) & saíra-amarela & $\mathrm{C}$ & $\mathrm{R}$ & \\
\hline Tersina viridis (Illiger, 1811) & saí-andorinha & $\mathrm{E}$ & M & \\
\hline Dacnis cayana (Linnaeus, 1766) & saí-azul & A & $\mathrm{R}$ & \\
\hline Hemithraupis ruficapilla (Vieillot, 1818) & saíra-ferrugem & $\mathrm{E}$ & $\mathrm{R}$ & ATL \\
\hline Hemithraupis flavicollis (Vieillot, 1818) C & saíra-galega & 1 & & \\
\hline Conirostrum speciosum (Temminck, 1824) & figuinha-de-rabo-castanho & $\mathrm{E}$ & $\mathrm{R}$ & \\
\hline \multicolumn{5}{|l|}{ Emberizidae } \\
\hline Zonotrichia capensis (Statius Muller, 1776) * & tico-tico & $A$ & $\mathrm{R}$ & \\
\hline Ammodramus humeralis (Bosc, 1792) C & tico-tico-do-campo & 1 & & \\
\hline Sicalis citrina Pelzeln, $1870 \mathrm{C}$ & canário-rasteiro & 1 & & \\
\hline Sicalis flaveola (Linnaeus, 1766) * AM & canário-da-terra-verdadeiro & $\mathrm{R}$ & $\mathrm{I}$ & \\
\hline Emberizoides herbicola (Vieillot, 1817) R & canário-do-campo & $\mathrm{O}$ & 1 & \\
\hline Embernagra platensis (Gmelin, 1789) C & sabiá-do-banhado & 1 & & \\
\hline Embernagra longicauda Strickland, $1844 \mathrm{C}$ & rabo-mole-da-serra & 1 & & \\
\hline Volatinia jacarina (Linnaeus, 1766) $*$ & tiziu & $\mathrm{R}$ & M & \\
\hline Sporophila lineola (Linnaeus, 1758) * \# & bigodinho & $\mathrm{O}$ & I & \\
\hline
\end{tabular}


Anexo I. Continuação.

\begin{tabular}{|c|c|c|c|c|}
\hline Nome do táxon & Nome popular & Abundância & Status migratório & Endemismo \\
\hline Sporophila nigricollis (Vieillot, 1823) * & baiano & C & 1 & \\
\hline Sporophila caerulescens (Vieillot, 1823) * \# & coleirinho & $\mathrm{O}$ & 1 & \\
\hline Sporophila albogularis (Spix, 1825) R*\# & golinho & 0 & I & \\
\hline Sporophila bouvreuil (Statius Muller, 1776) C & caboclinho & 1 & & \\
\hline Sporophila angolensis (Linnaeus, 1766) R* \#AM & curió & 0 & I & \\
\hline Arremon flavirostris Swainson, 1838 & tico-tico-de-bico-amarelo & $\mathrm{O}$ & I & \\
\hline Coryphospingus pileatus (Wied, 1821)* & tico-tico-rei-cinza & $\mathrm{R}$ & 1 & \\
\hline Paroaria dominicana (Linnaeus, 1758) C & cardeal-do-nordeste & 1 & & \\
\hline \multicolumn{5}{|l|}{ Cardinalidae } \\
\hline Saltator similis d'Orbigny \& Lafresnaye, $1837 *$ & trinca-ferro-verdadeiro & A & $\mathrm{R}$ & \\
\hline Saltator atricollis Vieillot, $1817 \mathrm{R} * \#$ & bico-de-pimenta & $\mathrm{O}$ & I & CSA \\
\hline Cyanocompsa brissonii (Lichtenstein, 1823) * & azulão & 0 & 1 & \\
\hline \multicolumn{5}{|l|}{ Parulidae } \\
\hline Geothlypis aequinoctialis (Gmelin, 1789) & pia-cobra & $\mathrm{R}$ & $\mathrm{R}$ & \\
\hline Basileuterus culicivorus (Deppe, 1830) & pula-pula & C & $\mathrm{R}$ & \\
\hline Basileuterus hypoleucus Bonaparte, 1830 & pula-pula-de-barriga-branca & C & $\mathrm{R}$ & \\
\hline Basileuterus flaveolus (Baird, 1865) R & canário-do-mato & A & $\mathrm{R}$ & \\
\hline \multicolumn{5}{|l|}{ Icteridae } \\
\hline Psarocolius decumanus (Pallas, 1769) & japu & $\mathrm{R}$ & $\mathrm{R}$ & \\
\hline Gnorimopsar chopi (Vieillot, 1819) & graúna & $\mathrm{R}$ & $\mathrm{R}$ & \\
\hline Chrysomus ruficapillus (Vieillot, 1819) * \# & garibaldi & $\mathrm{O}$ & $\mathrm{R}$ & \\
\hline M olothrus bonariensis (Gmelin, 1789) * & vira-bosta & $\mathrm{R}$ & 1 & \\
\hline Sturnella militaris (Linnaeus, 1758) C & polícia-inglesa-do-norte & 1 & & \\
\hline \multicolumn{5}{|l|}{ Fringillidae } \\
\hline Carduelis magellanica (Vieillot, 1805) C*\# & pintassilgo & $\mathrm{R}$ & & \\
\hline Euphonia chlorotica (Linnaeus, 1766) & fim-fim & A & $\mathrm{R}$ & \\
\hline Euphonia cyanocephala (Vieillot, 1818) C & gaturamo-rei & 1 & & \\
\hline \multicolumn{5}{|l|}{ Passeridae } \\
\hline Passer domesticus (Linnaeus, 1758) R & pardal & $\mathrm{R}$ & $\mathrm{R}$ & \\
\hline
\end{tabular}

